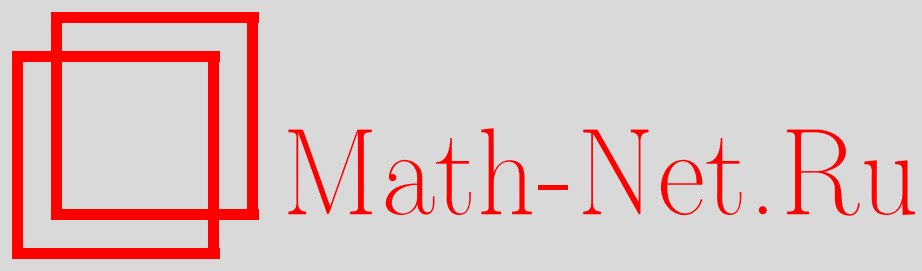

С. Дас, В. К. Ядав, Управление хаосом и функциональная проективная синхронизация систем дробного порядка методом бэкстеппинга, ТМФ, 2016, том 189, номер 1, 36-47

DOI: https://doi.org/10.4213/tmf9046

Использование Общероссийского математического портала Math-Net.Ru подразумевает, что вы прочитали и согласны с пользовательским соглашением http://www . mathnet.ru/rus/agreement

Параметры загрузки:

IP : 54.198 .187 .58

26 апреля 2023 г., $17: 26: 00$

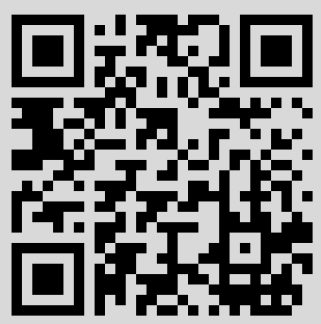




\title{
УПРАВЛЕНИЕ ХАОСОМ И ФУНКЦИОНАЛЬНАЯ ПРОЕКТИВНАЯ СИНХРОНИЗАЦИЯ СИСТЕМ ДРОБНОГО ПОРЯДКА МЕТОДОМ БЭКСТЕППИНГА
}

\begin{abstract}
C помощью метода бэкстеппинга изучаются проблема управления хаосом и функциональная проективная синхронизация между Т-системой дробного порядка и хаотической системой Лоренца. На основе теории устойчивости рассмотрено условие локальной устойчивости нелинейной трехмерной системы соизмеримого дробного порядка. Для управления хаосом в Т-системе дробного порядка использован метод обратной связи. Выполнено численное моделирование функциональной проективной синхронизации между Т-системой дробного порядка и системой Лоренца.
\end{abstract}

Ключевые слова: производная дробного порядка, хаотическая Т-система, система Лоренца, метод бэкстеппинга, метод обратной связи, теория устойчивости Ляпунова, синхронизация.

DOI: $10.4213 /$ tmf9046

\section{1. ВВЕДЕНИЕ}

Управление хаосом и хаотическая динамика систем дробного порядка являются важными направлениями исследований, особенно задачи управления и синхронизации хаоса в нелинейных динамических системах. Синхронизацией хаоса называется процесс, при котором происходит настройка двух идентичных или неидентичных хаотических систем с учетом свойств их движения, в результате которой они, взаимодействуя, начинают вести себя одинаково. Таким образом, возникает структурная устойчивость. Эти идеи мотивировали исследователей к созданию математических моделей синхронизации двух систем дробного порядка.

Преимущество систем дробного порядка состоит в том, что они позволяют получать более гибкие модели. Дифференциальный оператор дробного порядка нелокален в отличие от локального дифференциального оператора целого порядка в том смысле, что для дифференциального оператора дробного порядка будущее определяется не только текущим состоянием, но и всеми предыдущими состояниями.

${ }^{*}$ Department of Mathematical Sciences, Indian Institute of Technology (Banaras Hindu University), Varanasi, India. E-mail: sdas.apm@iitbhu.ac.in 
Из-за этого реалистичного свойства вычисления с производными дробного порядка, которые ранее рассматривались только как математическая диковина, теперь стали объектом интенсивного исследования, направленного на их дальнейшее применение в инженерных приложениях.

Функциональная проективная синхронизация - это обобщение проективной синхронизации, которая является одним из наиболее важных методов синхронизации и предметом широкого изучения с целью получения более быстрой связи путем использования свойств пропорциональности. При функциональной проективной синхронизации системы, описывающие возбуждение и отклик, синхронизованы с помощью масштабной функции. Очевидно, что присутствие масштабной функции в функциональной проективной синхронизации дополнительно повышает надежность сообщения. При этом многие методы функциональной проективной синхронизации сфокусированы лишь на хаотических системах целого порядка [1]-[5], а результатов, касающихся функциональной проективной синхронизации для хаотических систем дробного порядка, немного [6]-[8].

В настоящей статье мы изучаем динамическое поведение и управление хаосом в случае Т-системы дробного порядка. Показано, что в Т-системе существует хаотический аттрактор. Для анализа критериев устойчивости в Т-системе дробного порядка используются условия Рауса-Гурвица дробного порядка. Для управления хаосом в рассматриваемой системе получены условия на линейную обратную связь. Функциональная проективная синхронизация Т-системы дробного порядка и системы Лоренца осуществляется методом бэкстеппинга.

\section{2. ПРЕДВАРИТЕЛЬНЫЕ СВЕДЕНИЯ И УСЛОВИЕ УСТОЙЧИВОСТИ}

\section{1. Определения и лемма.}

ОПРЕДЕЛЕНиЕ 2.1. Производная Капуто [9] дробного порядка $q$ определяется KaK

$$
{ }_{t_{0}}^{\mathrm{C}} D_{t}^{q} \phi(t)=\frac{1}{\Gamma(n-q)} \int_{t_{0}}^{t} \frac{\phi^{(n)}(\tau)}{(t-\tau)^{q+1-n}} d \tau, \quad t>t_{0},
$$

для $n-1<q<n, n \in \mathbb{N}, \phi \in C_{-1}^{n}$.

ЗАмечАниЕ. Действительнозначная функция $\phi(t), t>t_{0}$, принадлежит пространству $C_{\alpha}^{n}$, если $\phi^{(n)} \in C_{\alpha}$ для $\alpha \in \mathbb{R}$, где $C_{\alpha}$ - пространство положительных действительнозначных функций таких, что существует действительное число $p>\alpha$, для которого $\phi(t)=t^{p} \phi_{1}(t)$ при $\phi_{1}(t) \in C\left[t_{0}, \infty\right]$.

В работе [10] доказана следующая лемма.

ЛЕмма 2.1. Пусть $x(t), t>0$, есть непрерьвная дифферениируемая действительнозначная функиия. Тогда в любой момент времени $t \geqslant t_{0}$

$$
\frac{1}{2}{ }_{t_{0}}^{\mathrm{C}} D_{t}^{q} x^{2}(t) \leqslant x(t){ }_{t_{0}}^{\mathrm{C}} D_{t}^{q} x(t)
$$

для всех $q \in(0,1)$. 
ОПРЕДЕЛЕНИЕ 2.2. [11]. Седловая точка индекса 1 - это точка равновесия системы, в которой якобиан имеет одно собственное значение с неотрицательной действительной частью (т. е. неустойчивое собственное значение). Седловая точка индекca 2 - это точка равновесия, в которой якобиан имеет два неустойчивых собственных значения.

Спиральные аттракторы возникают только вокруг седловых точек индекса 2. Седловые точки индекса 1 отвечают за соединение спиралей.

2.2. Устойчивость системы. Рассмотрим динамическую систему дробного порядка

$$
\frac{d^{q} x(t)}{d t^{q}}=f_{1}(x, y, z) \quad \frac{d^{q} y(t)}{d t^{q}}=f_{2}(x, y, z), \quad \frac{d^{q} z(t)}{d t^{q}}=f_{3}(x, y, z),
$$

где $q \in(0,1)$ и $\frac{d^{q}}{d t^{q}}={ }_{t_{0}}^{\mathrm{C}} D_{t}^{q}-$ производная Капуто. Якобиан системы в точках равновесия имеет вид

$$
J=\left[\begin{array}{lll}
\frac{\partial f_{1}}{\partial x} & \frac{\partial f_{1}}{\partial y} & \frac{\partial f_{1}}{\partial z} \\
\frac{\partial f_{2}}{\partial x} & \frac{\partial f_{2}}{\partial y} & \frac{\partial f_{2}}{\partial z} \\
\frac{\partial f_{3}}{\partial x} & \frac{\partial f_{3}}{\partial y} & \frac{\partial f_{3}}{\partial z}
\end{array}\right]
$$

Следующую теорему можно найти в работах [12], [13].

ТЕОрема 2.1. Система (1) локально асимптотически устойчива, если все собственные значения якобиана в точке равновесия удовлетворяют неравенству

$$
|\arg (\lambda)|>\frac{q \pi}{2}
$$

Характеристическое уравнение для якобиана (2) в точке равновесия записывается как

$$
P(\lambda)=\lambda^{3}+a_{1} \lambda^{2}+a_{2} \lambda+a_{3},
$$

а его дискриминант имеет вид

$$
D(P)=18 a_{1} a_{2} a_{3}+\left(a_{1} a_{2}\right)^{2}-4 a_{3} a_{1}^{3}-4 a_{2}^{3}-27 a_{3}^{2} .
$$

Условия Рауса-Гурвица [14], [15] для системы дробного порядка суть следующие условия:

a) если $D(P)>0$, то неравенства $a_{1}>0, a_{3}>0, a_{1} a_{2}-a_{3}>0$ дают необходимые и достаточные условия локальной асимптотической устойчивости точки равновесия;

б) если $D(P)<0$ и $a_{1} \geqslant 0, a_{2} \geqslant 0, a_{3}>0$, то точка равновесия системы локально асимптотически устойчива при $q<2 / 3$; если $D(P)<0$ и $a_{1}<0, a_{2}<0$, а $q>2 / 3$, то все корни уравнения (4) удовлетворяют условию $|\arg (\lambda)|<q \pi / 2$;

в) если $D(P)<0$ и $a_{1}>0, a_{2}>0, a_{1} a_{2}-a_{3}=0$, то точка равновесия локально асимптотически устойчива при всех $0<q<1$;

г) неравенство $a_{3}>0$ есть необходимое условие локальной асимптотической устойчивости точки равновесия. 


\section{3. ОПИСАНИЕ СИСТЕМЫ И ЕЕ УСТОЙЧИВОСТЬ}

3.1. Т-система дробного порядка. Хаотическая динамическая Т-система, представленная Тиганом [16], [17], имеет вид

$$
\frac{d x}{d t}=a(y-x), \quad \frac{d y}{d t}=(c-a) x-a x z, \quad \frac{d z}{d t}=-b z+x y,
$$

где $a, b, c$ - параметры и $x, y, z$ - переменные, описывающие состояние системы. Если выбрать $(a, b, c)=(2.1,0.6,30)$, то максимальный показатель Ляпунова равен 0.37 , и в Т-системе наблюдается хаос.

Т-система дробного порядка задается как

$$
\frac{d^{q} x}{d t^{q}}=a(y-x), \quad \frac{d^{q} y}{d t^{q}}=(c-a) x-a x z, \quad \frac{d^{q} z}{d t^{q}}=-b z+x y,
$$

где $0<q<1$. Рис. 1 показывает, что Т-система демонстрирует регулярное хаотическое поведение при дробном порядке $q=0.95$, если параметры выбраны как $(a, b, c)=(2.1,0.6,30)$, а начальные условия $(x(0), y(0), z(0))=(0.1,1.2,-0.5)$. На рис. 2 показаны траектории Т-системы при тех же значениях параметров и начальных условиях, но для $q=0.94$, чтобы подтвердить устойчивую природу траекторий.

3.2. Точки равновесия и устойчивость. Уравнения, определяющие точки равновесия системы (7), таковы:

$$
a(y-x)=0, \quad(c-a) x-a x z=0, \quad-b z+x y=0,
$$

и при заданных выше значениях параметров точки равновесия имеют координаты

$$
E_{1}=(0,0,0), \quad E_{2}=(2.8234,2.8234,13.2857), \quad E_{3}=(-2.8234,-2.8234,13.2857) .
$$

Якобиан системы $(7)$ в точке равновесия $\bar{E}=(\bar{x}, \bar{y}, \bar{z})$ задается как

$$
J(\bar{E})=\left[\begin{array}{ccc}
-a & a & 0 \\
c-a-a \bar{z} & 0 & -a \bar{x} \\
\bar{y} & \bar{x} & -b
\end{array}\right] .
$$

Его характеристический многочлен есть

$$
P(\lambda)=\lambda^{3}+2.7 \lambda^{2}+\left(2.7 \bar{x}^{2}+4.41 \bar{z}-57.33\right) \lambda+4.41 \bar{x}^{2}+4.41 \bar{x} \bar{y}+2.646 \bar{z}-35.154 .
$$

В точке равновесия $E_{1}=(0,0,0)$ уравнение $(10)$ записывается как

$$
P(\lambda)=\lambda^{3}+2.7 \lambda^{2}-57.33 \lambda-35.154 .
$$

Собственные значения суть $\lambda_{1}=-8.7761, \lambda_{2}=6.6761, \lambda_{3}=-0.60$. Видно, что точка равновесия $E_{0}$ является седловой точкой индекса 1 и по определению 2.2 она неустойчива при $0<q<1$. В точке равновесия $E_{2}=(2.8234,2.8234,13.2857)$ уравнение (10) записывается как

$$
P(\lambda)=\lambda^{3}+2.7 \lambda^{2}+18.0002 \lambda+70.3093,
$$

а собственные значения суть $\lambda_{1}=-3.4294, \lambda_{2,3}=0.3647 \pm 4.5132 i$, откуда следует, что точка равновесия $E_{2}$ является по определению 2.2 седловой точкой индекса 2 Таким образом, точка $E_{2}$ устойчива при $q<0.949$. Аналогично, точка равновесия $E_{3}$ устойчива при $q<0.949$. 

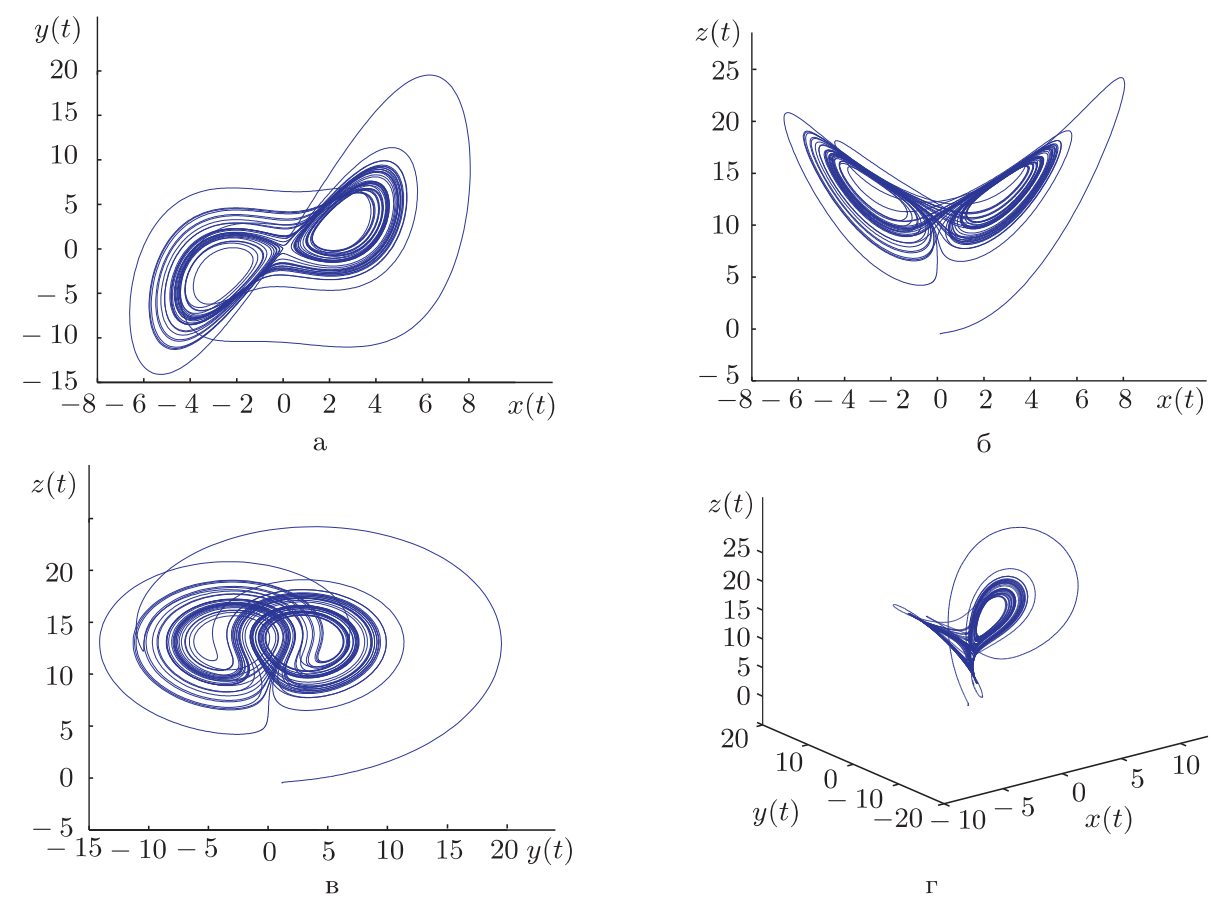

Рис. 1. Фазовые портреты Т-системы дробного порядка $q=0.95$.

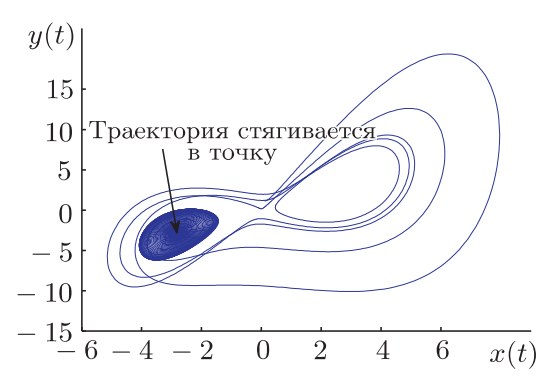

a

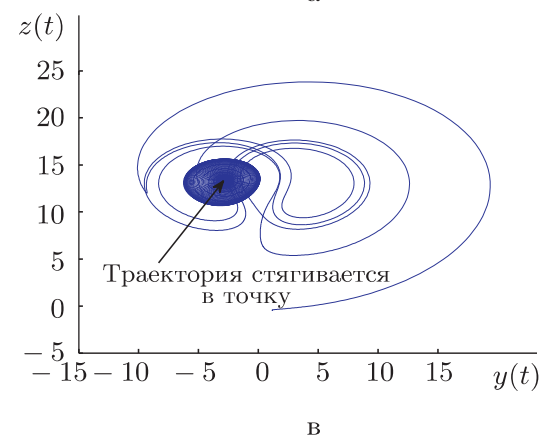

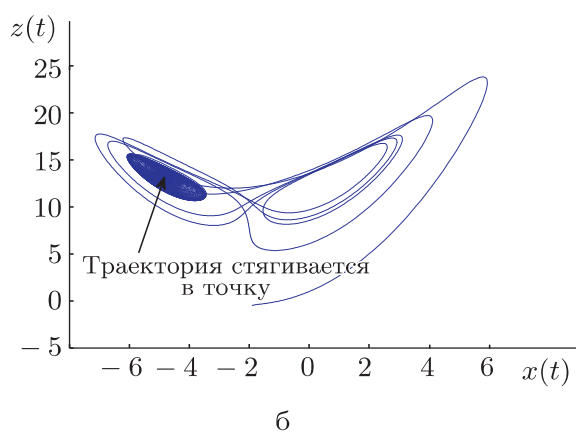

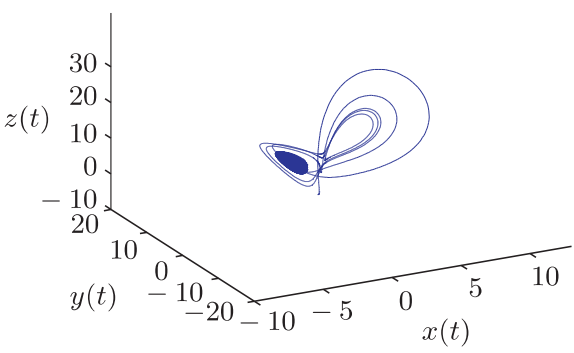

$\Gamma$

Рис. 2. Фазовые портреты Т-системы дробного порядка $q=0.94$. 


\section{4. УПРАВЛЕНИЕ ХАОСОМ}

Т-система дробного порядка с контроллером задана как

$$
\begin{aligned}
& \frac{d^{q} x}{d t^{q}}=a(y-x)-k_{1}(x-\bar{x}), \\
& \frac{d^{q} y}{d t^{q}}=(c-a) x-a x z-k_{2}(y-\bar{y}), \\
& \frac{d^{q} z}{d t^{q}}=-b z+x y-k_{3}(z-\bar{z}),
\end{aligned}
$$

где $k_{1}, k_{2}, k_{3}$ - управляющие параметры, а $(\bar{x}, \bar{y}, \bar{z})$ - точки равновесия. Якобиан этой системы в точке равновесия $\bar{E}=(\bar{x}, \bar{y}, \bar{z})$ имеет вид

$$
J(\bar{E})=\left[\begin{array}{ccc}
-a-k_{1} & a & 0 \\
c-a-a \bar{z} & -k_{2} & -a \bar{x} \\
\bar{y} & \bar{x} & -b-k_{3}
\end{array}\right] .
$$

При $a=2.1, b=0.6, c=30$ получаем соответствующий характеристический многочлен

$$
\begin{aligned}
P(\lambda)= & \lambda^{3}+\left(k_{1}+k_{2}+k_{3}+2.7\right) \lambda^{2}+ \\
& +\left[2.1 \bar{x}^{2}+4.41 \bar{z}+\left(k_{3}+0.6\right)\left(k_{1}+k_{2}+2.1\right)+k_{2}\left(k_{1}+2.1\right)-58.59\right] \lambda+ \\
& +\left[4.41 \bar{x} \bar{y}+2.1 \bar{x}^{2}\left(k_{1}+k_{2}+2.1\right)-2.1 k_{2} \bar{x}^{2}+\right. \\
& \left.\quad+\left(k_{3}+0.6\right)\left(4.41 \bar{z}+k_{2}\left(k_{1}+2.1\right)-58.59\right)\right] .
\end{aligned}
$$

Принимая во внимание условия Рауса-Гурвица дробного порядка, получаем

$$
\begin{aligned}
& a_{1}=k_{1}+k_{2}+k_{3}+2.7, \\
& a_{2}=2.1 \bar{x}^{2}+4.41 \bar{z}+\left(k_{3}+0.6\right)\left(k_{1}+k_{2}+2.1\right)+k_{2}\left(k_{1}+2.1\right)-58.59, \\
& a_{3}=4.41 \bar{x} \bar{y}+2.1 \bar{x}^{2}\left(k_{1}+k_{2}+2.1\right)-2.1 k_{2} \bar{x}^{2}+ \\
& \quad+\left(k_{3}+0.6\right)\left(4.41 \bar{z}+k_{2}\left(k_{1}+2.1\right)-58.59\right) .
\end{aligned}
$$

Устойчивость точек $E_{1}, E_{2}$ и $E_{3}$. Подставляя координаты точки $E_{1}$ в равенства (15) и принимая $k_{1}=4, k_{2}=10$ и $k_{3}=1$, имеем $D(P)>0, a_{1}>0, a_{3}>0$, $a_{1} a_{2}-a_{3}>0$. Все собственные значения, получающиеся из уравнения (14), действительные и отрицательные. Таким образом, система (13) локально асимптотически устойчива при $0<q<1$ (см. рис. 3a).

Подставляя координаты точки $E_{2}$ в равенства (15) и принимая $k_{1}=1, k_{2}=5$ и $k_{3}=0.6$, имеем $D(P)>0, a_{1}>0, a_{3}>0, a_{1} a_{2}-a_{3}>0$. Следовательно, все собственные значения, получающиеся из уравнения (14), действительные и отрицательные. Таким образом, система (13) локально асимптотически устойчива при $0<q<1$ (см. рис. 3б). 


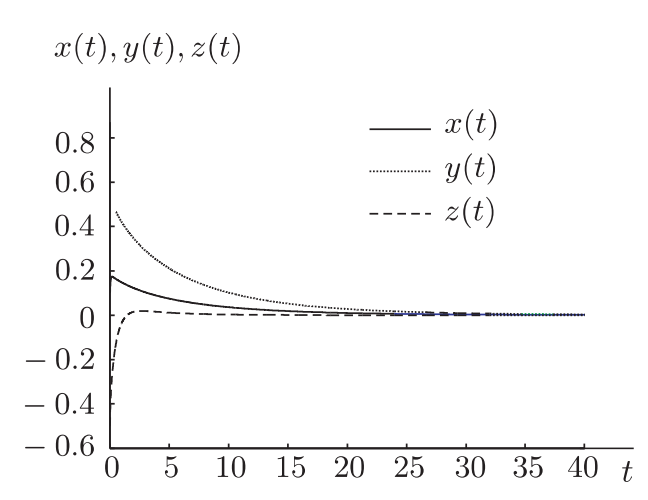

a
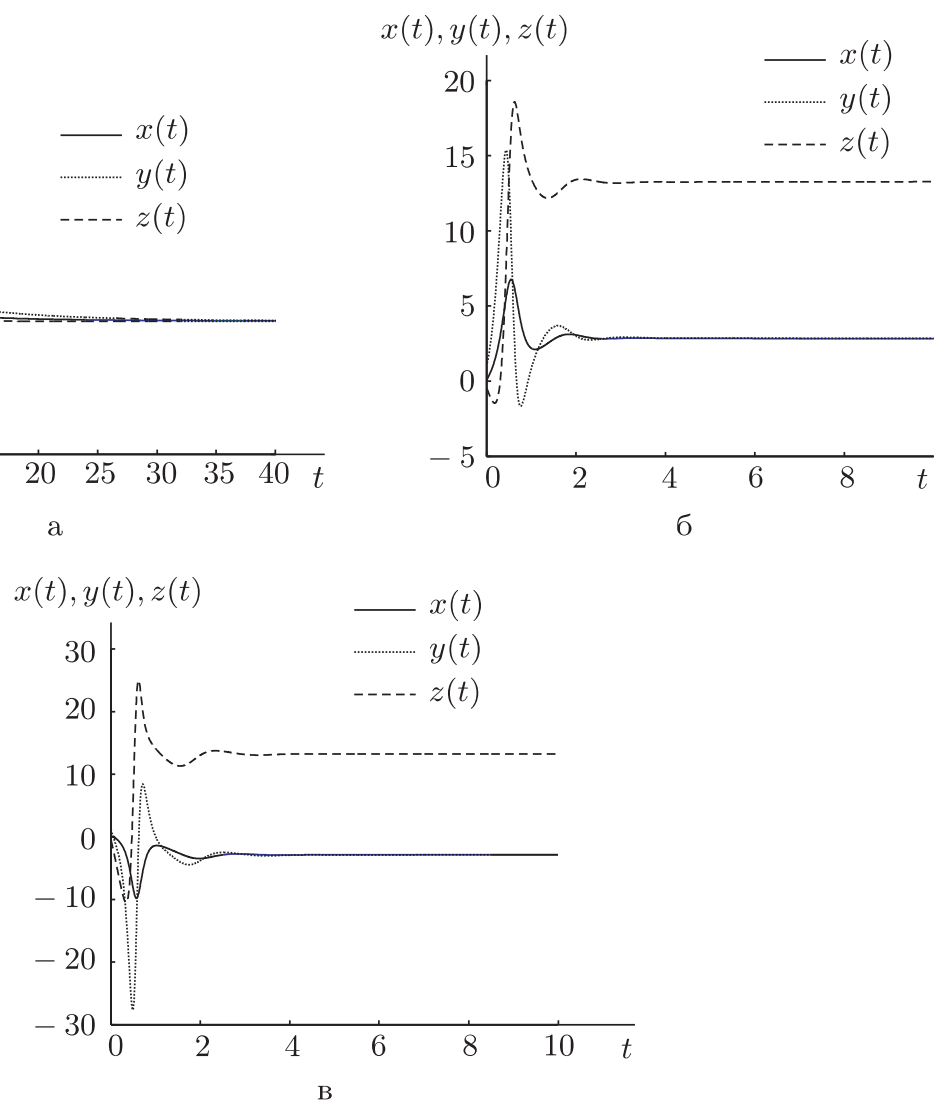

Рис. 3. Графики функций $x(t), y(t), z(t)$ для контролируемой системы (13) в точке равновесия $E_{1}(\mathrm{a})$, в точке равновесия $E_{2}$ (б) и в точке равновесия $E_{3}$ (в).

Аналогично, в точке равновесия $E_{3}$ при $k_{1}=1, k_{2}=6$ и $k_{3}=-2$ система (13) локально асимптотически устойчива при $0<q<1$ (см. рис. 3в).

\section{5. ФУНКЦИОНАЛЬНАЯ ПРОЕКТИВНАЯ СИНХРОНИЗАЦИЯ МЕЖДУ НЕИДЕНТИЧНЫМИ Т-СИСТЕМОЙ И СИСТЕМОЙ ЛОРЕНЦА ДРОБНЫХ ПОРЯДКОВ}

Система Лоренца дробного порядка задается как [18], [19]

$$
\frac{d^{q} x}{d t^{q}}=\alpha(y-x), \quad \frac{d^{q} y}{d t^{q}}=x(\gamma-z)-y, \quad \frac{d^{q} z}{d t^{q}}=x y-\beta z,
$$

где $\alpha$ - число Прандтля, $\gamma$ - число Рэлея, а параметр $\beta$ связан с геометрическим размером характерной области, заданной системой (16). Фазовый портрет системы Лоренца при $q=0.993$ показан на рис. 4; значения параметров выбраны как $\alpha=10$, $\beta=8 / 3, \gamma=28$, а координаты начальной точки - как $(0.1,0.1,0.1)$. 


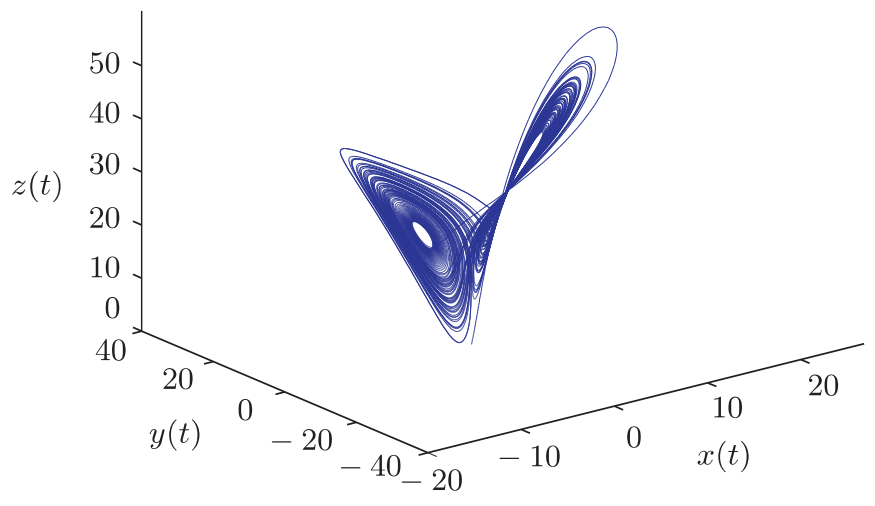

Рис. 4. Фазовый портрет системы Лоренца для порядка производной $q=0.993$.

Чтобы провести функциональную проективную синхронизацию между Т-системой и системой Лоренца дробных порядков, рассмотрим Т-систему в качестве управляющей системы, а систему Лоренца - в качестве подчиненной:

$$
\begin{aligned}
\frac{d^{q} x_{1}}{d t^{q}} & =a\left(y_{1}-x_{1}\right), & \frac{d^{q} x_{2}}{d t^{q}} & =\alpha\left(y_{2}-x_{2}\right)+u_{1}(t), \\
\frac{d^{q} y_{1}}{d t^{q}} & =(c-a) x_{1}-a x_{1} z_{1}, & \frac{d^{q} y_{2}}{d t^{q}} & =x_{2}\left(\gamma-z_{2}\right)-y_{2}+u_{2}(t), \\
\frac{d^{q} z_{1}}{d t^{q}} & =-b z_{1}+x_{1} y_{1}, & \frac{d^{q} z_{2}}{d t^{q}} & =x_{2} y_{2}-\beta z_{2}+u_{3}(t),
\end{aligned}
$$

где $u_{1}(t), u_{2}(t), u_{3}(t)$ - управляющие функции.

Введем функции $e_{1}=x_{2}-m_{1} x_{1}, e_{2}=y_{2}-m_{2} y_{1}$ и $e_{3}=z_{2}-m_{3} z_{1}$, характеризующие отклонения решений подчиненной и управляющей систем друг от друга; здесь $m_{1}$, $m_{2}$ и $m_{3}$ - масштабные функции. В терминах отклонений динамическая система принимает вид

$$
\begin{aligned}
& \frac{d^{q} e_{1}}{d t^{q}}=\alpha\left(e_{2}-e_{1}\right)+\psi_{1}+u_{1}(t), \\
& \frac{d^{q} e_{2}}{d t^{q}}=e_{1}\left(\gamma-e_{3}\right)-e_{1} m_{3} z_{1}-e_{3} m_{1} x_{1}-e_{2}+\psi_{2}+u_{2}(t), \\
& \frac{d^{q} e_{3}}{d t^{q}}=e_{1} e_{2}+e_{1} m_{2} y_{1}+e_{2} m_{1} x_{1}-\beta e_{3}+\psi_{3}+u_{3}(t),
\end{aligned}
$$

где

$$
\begin{aligned}
& \psi_{1}=\alpha m_{2} y_{1}-\alpha m_{1} x_{1}-m_{1} a\left(y_{1}-x_{1}\right), \\
& \psi_{2}=m_{1} \gamma x_{1}-m_{1} m_{3} z_{1} x_{1}-m_{2}\left[y_{1}+(c-a) x_{1}-a x_{1} z_{1}\right], \\
& \psi_{3}=m_{1} m_{2} x_{1} y_{1}-m_{3}\left[(\beta-b) z_{1}+x_{1} y_{1}\right] .
\end{aligned}
$$

ШАГ 1. Пусть $w_{1}=e_{1}$, тогда производная дробного порядка от $w_{1}$ имеет вид

$$
\frac{d^{q} w_{1}}{d t^{q}}=\frac{d^{q} e_{1}}{d t^{q}}=\alpha\left(e_{2}-w_{1}\right)+\psi_{1}+u_{1}(t)
$$

где $e_{2}=\alpha_{1}\left(w_{1}\right)$ - виртуальный контроллер. Для стабилизации подсистемы $w_{1}$ определим функцию Ляпунова $V_{1}=w_{1}^{2} / 2$. Мы можем оценить ее дробную производную 
$q$-го порядка по $t:$

$$
\frac{d^{q} V_{1}}{d t^{q}}=\frac{1}{2} \frac{d^{q} w_{1}^{2}}{d t^{q}} \leqslant w_{1} \frac{d^{q} w_{1}}{d t^{q}} \leqslant w_{1}\left[\alpha\left(\alpha_{1}\left(w_{1}\right)-w_{1}\right)+\psi_{1}+u_{1}(t)\right]
$$

где первое неравенство получено с учетом леммы 2.1. Положив

$$
\alpha_{1}\left(w_{1}\right)=w_{1}-\frac{w_{1}}{\alpha}, \quad u_{1}(t)=-\psi_{1},
$$

получаем, что производная $d^{q} V_{1} / d t^{q} \leqslant-w_{1}^{2}<0$, откуда следует, что подсистема (20) асимптотически устойчива.

Для виртуальной управляющей функции $\alpha_{1}\left(w_{1}\right)$ введем функцию $w_{2}=e_{2}-$ $\alpha_{1}\left(w_{1}\right)$, связывающую $e_{2}$ и $\alpha_{1}\left(w_{1}\right)$. Тогда подсистема для функций $\left(w_{1}, w_{2}\right)$ записывается как

$$
\begin{aligned}
& \frac{d^{q} w_{1}}{d t^{q}}=\alpha w_{2}-w_{1} \\
& \frac{d^{q} w_{2}}{d t^{q}}=-\alpha w_{2}+w_{1}\left(\gamma-e_{3}\right)-w_{1} m_{3} z_{1}-e_{3} m_{1} x_{1}+\psi_{2}+u_{2}(t)
\end{aligned}
$$

где $e_{3}=\alpha_{2}\left(w_{1}, w_{2}\right)$ можно рассматривать как виртуальный контроллер.

ШАГ 2. На данном шаге, чтобы стабилизировать подсистему (21), определим функцию Ляпунова

$$
V_{2}=V_{1}+\frac{1}{2} w_{2}^{2}=\frac{1}{2} w_{1}^{2}+\frac{1}{2} w_{2}^{2}
$$

Теперь имеем

$$
\begin{aligned}
\frac{d^{q} V_{2}}{d t^{q}}=\frac{1}{2} \frac{d^{q} w_{1}^{2}}{d t^{q}}+\frac{1}{2} \frac{d^{q} w_{2}^{2}}{d t^{q}} & \leqslant w_{1} \frac{d^{q} w_{1}}{d t^{q}}+w_{2} \frac{d^{q} w_{2}}{d t^{q}} \leqslant \\
& \leqslant-w_{1}^{2}-\alpha w_{2}^{2}+w_{2}\left[\alpha w_{1}+w_{1}\left(\gamma-\alpha_{2}\left(w_{1}, w_{2}\right)\right)-w_{1} m_{3} z_{1}-\right. \\
& \left.-\alpha_{2}\left(w_{1}, w_{2}\right) m_{1} x_{1}+\psi_{2}+u_{2}(t)\right]
\end{aligned}
$$

где мы применили сначала лемму 2.1, а затем уравнения (21). Если

$$
\alpha_{2}\left(w_{1}, w_{2}\right)=0, \quad u_{2}(t)=-\psi_{2}+w_{1} m_{3} z_{1}-w_{1} \gamma-\alpha w_{1},
$$

то в силу неравенства $d^{q} V_{2} / d t^{q} \leqslant-w_{1}^{2}-\alpha w_{2}^{2}<0$ система (21) становится асимптотически устойчивой.

Положив $w_{3}=e_{3}-\alpha_{2}\left(w_{1}, w_{2}\right)$, получаем следующую подсистему уравнений относительно $\left(w_{1}, w_{2}, w_{3}\right)$ :

$$
\begin{aligned}
& \frac{d^{q} w_{1}}{d t^{q}}=\alpha w_{2}-w_{1} \\
& \frac{d^{q} w_{2}}{d t^{q}}=-\alpha\left(w_{1}+w_{2}\right)-w_{1} w_{3}-w_{3} m_{1} x_{1} \\
& \frac{d^{q} w_{3}}{d t^{q}}=w_{1}\left(w_{2}+w_{1}-\frac{w_{1}}{\alpha}\right)+w_{1} m_{2} y_{1}+\left(w_{2}+w_{1}-\frac{w_{1}}{\alpha}\right) m_{1} x_{1}-\beta w_{3}+\psi_{3}+u_{3}(t) .
\end{aligned}
$$


ШАГ 3. Для стабилизации подсистемы (22) выберем фунцию Ляпунова в виде

$$
V_{3}=V_{2}+\frac{1}{2} w_{3}^{2}=\frac{1}{2} w_{1}^{2}+\frac{1}{2} w_{2}^{2}+\frac{1}{2} w_{3}^{2}
$$

и получим, вновь используя лемму 2.1 ,

$$
\begin{aligned}
\frac{d^{q} V_{3}}{d t^{q}}= & \frac{1}{2} \frac{d^{q} w_{1}^{2}}{d t^{q}}+\frac{1}{2} \frac{d^{q} w_{2}^{2}}{d t^{q}}+\frac{1}{2} \frac{d^{q} w_{3}^{2}}{d t^{q}} \leqslant w_{1} \frac{d^{q} w_{1}}{d t^{q}}+w_{2} \frac{d^{q} w_{2}}{d t^{q}}+w_{3} \frac{d^{q} w_{3}}{d t^{q}} \leqslant \\
\leqslant & -w_{1}^{2}-\alpha w_{2}^{2}-\beta w_{3}^{2}+w_{3}\left[-w_{1}-w_{3} m_{1} x_{1}+\right. \\
& \left.+w_{1}\left(w_{2}+w_{1}-\frac{w_{1}}{\alpha}\right)+w_{1} m_{2} y_{1}+\left(w_{2}+w_{1}-\frac{w_{1}}{\alpha}\right) m_{1} x_{1}+\psi_{3}+u_{3}(t)\right] .
\end{aligned}
$$

Если

$u_{3}(t)=-\psi_{3}-\left(w_{2}+w_{1}-\frac{w_{1}}{\alpha}\right) m_{1} x_{1}+w_{3} m_{1} x_{1}-w_{1}\left(w_{2}+w_{1}-\frac{w_{1}}{\alpha}\right)-w_{1} m_{2} y_{1}+w_{1}$,

то

$$
\frac{d^{q} V_{3}}{d t^{q}} \leqslant-w_{1}^{2}-\alpha w_{2}^{2}-\beta w_{3}^{2}<0
$$

С учетом выражений

$$
w_{1}=e_{1}, \quad w_{2}=e_{2}-\alpha_{1}\left(w_{1}\right)=e_{2}-e_{1}+\frac{e_{1}}{\alpha}, \quad w_{3}=e_{2}-\alpha_{2}\left(w_{1}, w_{2}\right)=e_{3}
$$

заключаем, что отклонения $e_{1}, e_{2}$ и $e_{3}$ становятся равными нулю за конечное время, следовательно, функциональная прективная синхронизация между Т-системой дробного порядка и системой Лоренца установлена.

Численное моделирование и его результаты. При численном моделировании с помощью системы Matlab начальные данные управляющей системы и подчиненной системы были выбраны следующим образом:

$$
\left(x_{1}(0), y_{1}(0), z_{1}(0)\right)=(0.1,1.2,-0.5), \quad\left(x_{2}(0), y_{2}(0), z_{2}(0)\right)=(0.1,0.1,0.1) .
$$

Следовательно, $\left(e_{1}(0), e_{2}(0), e_{3}(0)\right)=(0,-1.1,0.6)$. Шаг по времени равен 0.005 . В качестве масштабных функций были выбраны

$m_{1}=a_{11} \cos \left(a_{12} x_{1}\right)+a_{13}, \quad m_{2}=a_{21} \cos \left(a_{22} y_{1}\right)+a_{23}, \quad m_{3}=a_{31} \cos \left(a_{32} z_{1}\right)+a_{33}$

с параметрами

$$
\begin{array}{lll}
a_{11}=0.5, & a_{12}=0.2, & a_{13}=0.1, \\
a_{21}=0.1, & a_{22}=0.3, & a_{23}=0.2, \\
a_{31}=0.2, & a_{32}=0.1, & a_{33}=0.3 .
\end{array}
$$




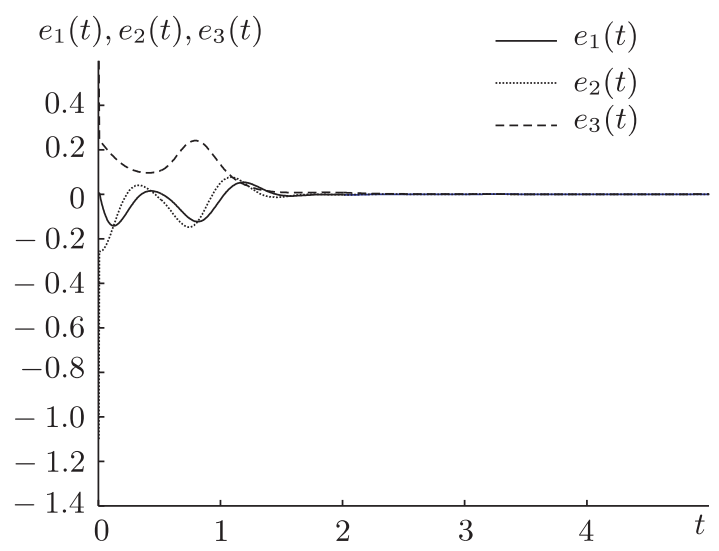

Рис. 5. Траектории отклонений $e_{1}(t), e_{2}(t)$ и $e_{3}(t)$ для управляющей системы (17) и подчиненной системы (18) при дробном порядке $q=0.993$.

На рис. 5 видно, что для $q=0.993$ отклонения асимптотически стремятся к нулю при больших временах, и это показывает, что управляющая система (17) синхронна с подчиненной системой (18).

\section{6. ВЫВОДЫ}

В представленной статье мы решили три важные задачи. Во-первых, была проанализирована локальная устойчивость Т-системы с производной по времени дробного порядка. Во-вторых, мы применили управляющую функцию Т-системы дробного порядка к различным точкам равновесия. С помощью дробного критерия Рауса-Гурвица с помощью линейного принципа обратной связи дробного порядка мы изучили устойчивость точек равновесия и достаточные условия для управления Т-системой дробного порядка. Мы заметили, что Т-система дробного порядка поддается управлению всюду, кроме точек равновесия. Теоремы устойчивости для систем дробного порядка утверждают, что управление хаосом можно осуществить, если выполнены некоторые необходимые условия. Результаты моделирования показывают, что управление с помощью обратной связи легко применить даже в случае хаотических систем дробного порядка. В-третьих, мы показали, что для функциональной проективной синхронизации Т-системы дробного порядка и системы Лоренца успешно применяется метод бэкстеппинга.

Благодарности. Авторы выражают свою искреннюю благодарность рецензентам за ценные замечания, направленные на улучшение статьи.

\section{Список литературы}

[1] Y. Chen, X. Li, Internat. J. Modern Phys. C, 18:5 (2007), 883-888.

[2] O. S. Ojoniyi, Int. J. Comp. Science, Eng. Inform. Technology (IJCSEIT), 4:5 (2014), 33-39.

[3] H. Du, Q. Zeng, C. Wang, M. Ling, Nonlinear Anal. Real World Appl., 11:2 (2010), 705-712.

[4] H. Du, Q. Zeng, C. Wang, Phys. Lett. A, 372:33 (2008), 5402-5410.

[5] C.-L. Zhang, J.-M. Li, Int. J. Automat. Comput., 9:4 (2012), 388-394. 
[6] P. Zhou, W. Zhu, Nonlinear Anal. Real World Appl., 12:2 (2011), 811-816.

[7] Z. Ping, C. Yu-Xia, Chinese Phys. B, 19:10 (2010), 100507.

[8] S. K. Agrawal, S. Das, J. Process Control, 24:5 (2014), 517-530.

[9] A. Kilbas, H. M. Srivastava, J. J. Trujillo, Theory and Applications of Fractional Differential Tquations, North-Holland Mathematics Studies, 204, Elsevier, Amsterdam, 2006.

[10] N. Aguila-Camacho, M. A. Duarte-Mermoud, J. A. Gallegos, Commun. Nonlinear Sci. Numer. Simul., 19:9 (2014), 2951-2957.

[11] M. R. Faieghi, H. Delavari, Commun. Nonlinear Sci. Numer. Simul., 17:2 (2012), 731-741.

[12] D. Matignon, "Stability results for fractional differential equations with applications to control processing", Computational Engineering in Systems and Application CESA'96 (Lille, France, July 9-12, 1996), 2, ed. P. Borne, GERF, Ecole Centrale de Lille, Villeneuve-d'Ascq, 1996, 963-968.

[13] C. Li, Y. Ma, Nonlinear Dynam., 71:4 (2013), 621-633.

[14] M. Srivastava, S. K. Agrawal, K. Vishal, S. Das, Appl. Math. Model., 38:13 (2014), 3361-3372.

[15] E. Ahmed, A. M. A. El-Sayed, H. A. A. El-Saka, Phys. Lett. A, 358:1 (2006), 1-4.

[16] G. Tigan, Bul. Ştiinţ. Univ. Politeh. Timiş. Ser. Mat. Fiz., 50(64):1 (2005), 61-72.

[17] G. Tigan, "Bifurcation and the stability in a system derived from the Lorenz system", Proceedings of the Third International Colloquium "Mathematics in Engineering and Numerical Physics" (Bucharest, Romania, October 7-9, 2004), Balkan Society of Geometers, Geometry Balkan Press, Bucharest, 2005, 265-272.

[18] X.-J. Wu, S.-L. Shen, Int. J. Comput. Math., 86:7 (2009), 1274-1282.

[19] I. Grigorenko, E. Grigorenko, Phys. Rev. Lett., 91:3 (2003), 034101, 4 pp. 